\title{
Attentional control in depression: A translational affective neuroscience approach
}

\author{
Rudi De Raedt ANd ERnst H. W. Koster \\ Ghent University, Ghent, Belgium \\ AND \\ JUTTA JOORMANN \\ University of Miami, Coral Gables, Florida
}

\begin{abstract}
Translational research refers to the application of basic science to address clinical problems and acquire knowledge that can be used to guide and refine clinical practice. This special issue of Cognitive, Affective, \& Behavioral Neuroscience seeks to explore and integrate some of the most promising findings offered by recent cognitive and affective neuroscience studies in hopes of filling the gap between basic and applied research, thereby heightening our understanding of vulnerability for depression. The studies presented in this special issue focus specifically on attentional processes. We solicited contributions from leading researchers involved in basic cognitive and neuroscience research investigating processes underlying depression-related disturbances in emotion processing. In this introductory article, we present an integrative overview to demonstrate how these specific contributions might be valuable for translational research.
\end{abstract}

Depression is a common and severe psychiatric illness that, despite immense research efforts, poses important challenges for prevention and treatment. Although many psychotherapeutic and pharmacological treatments have been developed, recent meta-analyses report rather small effect sizes (Cuijpers, van Straten, Bohlmeijer, Hollon, \& Andersson, in press; Kirsch et al., 2008; Turner, Matthews, Linardatos, Tell, \& Rosenthal, 2008). Moreover, within the group of depressed individuals who do show initial improvement in response to treatment, a large proportion experience relapse within a number of years after treatment completion, and depression becomes a recurrent problem (Goodwin, Jacobi, Bittner, \& Wittchen, 2006). These disappointing results provide an important impetus for researchers and clinicians to join forces to try to better understand and treat this debilitating disorder. Indeed, it might be crucial to rethink our current approaches to understanding and treating depression by integrating basic and clinical research and refining interventions accordingly.

Depression is a mood disorder characterized by sustained negative affect and loss of positive affect. First onsets of depression are frequently linked to the experience of stressful life events. Integrating basic research on biological stress reactivity and cognition, as well as that on the relation of cognitive processes to stress recovery, affect generation, and regulation, therefore, promises to improve our understanding and treatment of this debilitating disorder. Contemporary research on the interaction of cognition and emotion, often from an affective neuroscience perspective, has provided important insights into the functioning of normative as well as disrupted emotion networks in the brain. Recently, models have been developed that elucidate the manner in which affect impinges on the attentional control system and vice versa (Taylor \& Fragopanagos, 2005). These studies have helped to improve our understanding of the role of attention engagement and disengagement and other forms of cognitive control in emotion generation and regulation. It is important to note that these models have helped to identify cognitive processes, often operating on an automatic level, that may play a critical role in the generation of affective responses and impair people's ability to repair the ensuing affect. This research has also helped to identify brain regions and networks associated with emotion processing and cognitive control and the relation of these brain regions to other biological systems critical to regulating stress responding, such as the hypothalamic-pituitaryadrenal (HPA) axis. Basic science research on the interaction of cognition and emotion, therefore, may help to improve our understanding of individual differences in the ability to disengage from the elaborative processing of negative cognitions after confrontation with stressors, a core problem in depression.

Contemporary research on the interaction of cognition and emotion has also moved beyond merely demonstrating that affective states and traits are associated with biased cognitive processing, to investigating the causal 
mechanisms underlying affect regulation and emotional vulnerability, an important step in exploring possibilities for modification (for a recent review, see Mathews $\&$ MacLeod, 2005). Brain imaging studies, for example, have outlined changes in neural response patterns, such as increased activations in limbic regions and reduced activations in prefrontal cortex, that seem to be present in high-risk individuals even before the first episode of depression is experienced (e.g., Monk et al., 2008). Our most effective interventions, such as cognitive-behavioral therapy (CBT), focus on modifying maladaptive cognitions and cognitive biases. Remarkably, however, these interventions have undergone few modifications in the last 50 years, and the theoretical foundations of CBT for depression are still largely rooted in Beck's cognitive theory proposed in the 1960s (e.g., Beck, 1963, 1976).

Over the past few decades, we have seen an impressive increase in experimental psychopathology research, which has led to important improvements in our models of depression and in our thinking about biological and psychological factors that increase the risk for the onset of this disorder and play a critical role in the maintenance of depressive episodes (Beck, 2008). These findings, however, have not been translated into more efficient treatments.

We therefore consider the interplay between basic research and clinical research to be of crucial importance in improving our understanding of depression and its treatment. An important goal of this special issue of Cognition, Affective, \& Behavioral Neuroscience is to bring together some of the key developments in basic science on cognition and emotion and discuss their relevance to the understanding and treatment of depressive disorders. For this purpose, we will use a translational affective neuroscience approach.

\section{Translational Affective Neuroscience}

Translational research refers to the application of basic science to address clinical problems and acquire knowledge that can be used to guide and refine clinical practice. This special issue of Cognition, Affective, \& Behavioral Neuroscience seeks to explore and integrate some of the most promising findings offered by new studies to fill the gap between basic and applied research, thereby heightening our understanding of vulnerability for depression and ultimately improving treatment and prevention. To that end, we solicited contributions from leading researchers who are involved in basic cognitive and affective neuroscience research.

This special issue focuses specifically on attentional problems in depression. In recent years, evidence has been accumulated to suggest that specific attentional problems are observed when depressed and dysphoric people process affective material (Gotlib, Krasnoperova, Yue, \& Joormann, 2004; Leyman, De Raedt, Schacht, \& Koster, 2007). Specifically, depression is associated with difficulties in disengaging attention from mood-congruent material and with difficulties in terminating elaborative processing of such material. Given the crucial role of attention in general cognitive functioning, such as execu- tive control (Engle, 2002) and working memory (Hasher \& Zacks, 1979), as well as emotion-relevant functioning, such as emotional reactivity (MacLeod, Rutherford, Campbell, Ebsworthy, \& Holker, 2002), emotion regulation (Koole, 2009) and depressive symptoms (Beevers \& Carver, 2003), it seems warranted to further examine attentional factors in depression. This special issue presents an overview of basic research from different perspectives on attentional control that can provide crucial input to the development of innovative treatment and prevention strategies.

Numerous aspects of intervention could profit from affective neuroscience insights into the mechanisms underlying the generation and regulation of affect. Specifically, a better understanding of affect generation and regulation and of cognitive and neural mechanisms underlying pharmacological and psychological treatments could help (1) integrate these interventions in a theory-based way, (2) refine intervention strategies, (3) match patients to specific interventions, (4) tackle the problem of nonresponse to pharmacological or psychological treatments, and (5) predict and prevent relapse after initially successful treatments.

To facilitate a translational affective neuroscience approach, several lines of research are required, including, but not limited to, affective neuroscience research investigating the processes involved in depression-related disturbances in emotion processing and the influence of these disturbances on emotional reactivity, emotion regulation, and depressive symptoms. Moreover, integrative theoretical models are needed to help understand the emerging findings and to guide future research. In what follows, we present an overview of how the specific contributions in this special issue might be valuable for translational research.

\section{Processes Underlying Vulnerability for Depression}

A first requirement for understanding the nature of disrupted attentional and biological processes in depression is to obtain a detailed picture of the interplay between emotion processing and attention in the brain. The amygdala is a limbic structure that is a central part of many neural models of emotion and that has also been implicated in depression risk, but its role in emotion generation and regulation remains open to debate. Using sophisticated methodology, Pourtois, Spinelli, Seeck, and Vuilleumier (2010) recorded intracranial local field potentials from the intact left lateral amygdala in an epileptic patient in order to investigate neural responses to fearful faces presented in attended or unattended locations. These authors demonstrated an early effect of the presentation of emotional material in the left amygdala prior to, and independent of, attentional modulation. When attention was directed toward or away from fearful faces, they observed modulation of differential emotional responses at later stages of processing, suggesting separate effects of emotion and attention on amygdala activation. These results underscore the critical role of the amygdala in emotion processing - that is, 
responsible for detecting, generating, and maintaining emotions (Phan, Wager, Taylor, \& Liberzon, 2004) — and suggest that this structure is important in early and later stages of the processing of emotional material. Further support for a close relation among attentional processing of emotional material and variations in neurobiological systems comes from an attention paradigm used by Ellenbogen, Carson, and Pishva (2010). Attentional shifting during trials in which masked angry faces were presented predicted changes in cortisol release during a stress induction, suggesting that early automatic processing of emotional stimuli is related to the regulation of the HPA axis stress response. Taken together, these data are indicative of a close relation between the physiological stress response and left amygdala activity during the processing of threatening information.

Closely related to findings from these basic science studies, ever more evidence shows that a history of early exposure to stressors, such as childhood neglect and attachment problems, may contribute to changes in neurobiological systems, such as the HPA axis, brain structure and function (e.g., the hippocampus), and neurotransmitter systems, causing an increased susceptibility to affective disorders (e.g., Gerra et al., 2009). The relation between this increased neurobiological vulnerability and depression might be mediated by dysfunctional emotion processing. Indeed, childhood neglect has been shown to influence emotion processing in a negative way (e.g., Masten et al., 2008). Maheu et al. (2010) integrated research on cognitive and biological vulnerability in an fMRI study investigating whether specific brain dysfunctions could underlie the relation between exposure to early stressors and sensitivity to threat cues. Youths with a history of early caregiver deprivation and emotional neglect demonstrated significantly greater left amygdala and left anterior hippocampus activation during the processing of threatening stimuli. These findings suggest a specific scarring effect in limbic structures that may result from early exposure to stressors and may set the stage for increased vulnerability to emotional disorders.

Implications of these observations for translational research are nicely illustrated in the review by Browning, Holmes, and Harmer (2010). These authors discuss evidence that attentional biases are responsive to the administration of pharmacological agents in nonclinical populations. Although studies (e.g., Fu et al., 2004) involving clinical populations may indicate that the findings are secondary to improved clinical status rather than being a treatment effect, Browning et al.'s literature review suggests that pharmacological interventions affect an initial bias operating at an early stage of the processing of emotional material potentially related to an amygdala-based stimulus appraisal system. In contrast, psychological interventions affect attention at later stages of cognitive processing related to anterior cingulate cortex (ACC) and lateral prefrontal cortex (PFC) activity. Taken together, results obtained from these reviews and results obtained from the previously discussed experimental data showing differences in attention biases between early and late processing stages in depression can set the stage for a better understanding of how combined psychological and pharmacological treatments work. Thus, these findings can lead to the development of a theory-based way of integrating these interventions, or maybe even to the assignment of patients to specific forms of treatment.

The results of Browning et al.'s (2010) review article line up nicely with neural models of depression that suggest that the interaction between subcortical (amygdala) and cortical (prefrontal) processing is crucial to our understanding and treatment of depression. A large body of work on the function of the ACC has demonstrated that it may play an important role in emotional regulation and thus may be highly relevant for understanding the development of depression.

In particular, the ACC is an important brain region to examine in studies on the interaction of subcortical and cortical functioning. In a seminal article, Bush, Luu, and Posner (2000) proposed that conflict monitoring in the ACC can be conceived as a bridge between subcortical emotion processing and cortical attentional control. Pereira et al. (2010) used fMRI during a target detection task that followed the presentation of unpleasant versus neutral pictures to investigate neural correlates of behavioral interference from emotional stimuli. The results show that emotional context modulated brain responses. Specifically, the midcingulate cortex was recruited when participants performed target detection trials during the unpleasant context, and neural responses in this region mirrored the pattern of interference observed in the behavioral data. The authors conclude that the midcingulate cortex may be involved in the interaction between affective and motor signals in the brain and may be related to defensive responses. Thus, the cingulate cortex, which has been related to the signaling of the need for attentional control upon encountering emotionally salient stimuli, might also be related to exerting behavioral control on the environment.

Whereas many depression theories have focused on the processing of negative material, the processing of positive, rewarding stimuli may be crucial to our understanding of emotion regulation and stress reactivity in depressed and nondepressed participants. Indeed, a wealth of research has investigated processing of positive information in these groups, with the depressed participants showing marked disturbances in reward processing. These disturbances may be related to negative expectations about their ability to change events (hopelessness) causing reduced initiation of behavioral control in an effort to influence the environment, resulting in a decrease in rewarding responses from that environment. The specific role of reward sensitivity in depression was investigated by Holmes and Pizzagalli (2010), using event-related potentials during a task with no-incentive and reward conditions. These authors focused on action monitoring, defined as the ability to coordinate actions to maximize the likelihood of goal achievement, even following unexpected environmental changes. Previous studies have reported action monitoring dysfunction - particularly, disrupted error processing (Holmes \& Pizzagalli, 2008) - and decreased approachrelated behavior in depressed patients (for a review, see 
Pizzagalli, Dillon, Bogdan, \& Holmes, in press). Thus, impairments in action monitoring might be partially explained by abnormalities in incentive processing. First, the results were indicative of a generally heightened reactivity in early (automatic) stages of error processing in depression, unrelated to reward processing. Second, when rewards were available at later stages of information processing, depressed patients showed blunted responses, which are assumed to relate to conscious error awareness and subjective affective evaluative responses. As discussed previously, dysfunctional cognitive processing at later stages of information processing reliably emerge in depression. In fact, the specific psychophysiological patterns observed in these studies could be related to increased paralimbic activation and to a failure to recruit PFC-based cognitive control, possibly due to abnormal frontocingulate connectivity. Indeed, depression has been conceptualized as a failure of dorsal areas (related to cognitive control) to regulate ventral emotion-producing brain systems (Phillips, Drevets, Rauch, \& Lane, 2003).

The importance of dysfunctional reward processing in depression and the relation with attentional and behavioral control systems in the brain may underlie the successful use of behavioral activation as a treatment strategy for depression that aims to increase environmental reinforcement and reduce punishment. This therapeutic technique facilitates new rewarding experiences that may enhance incentive processing. Several studies have shown the effectiveness of behavioral activation. Indeed, a recent large-scale study demonstrated a better treatment response to behavioral activation than to cognitive therapy (Dimidjian et al., 2006).

The important role of the PFC in this neural attention/ emotion circuitry was addressed by Engels et al. (2010), who measured brain activity using fMRI during an emotion-word Stroop task, disentangling the influence of anxiety and depression, which are highly comorbid conditions. They found a right-sided asymmetrical lateralization pattern of PFC activity for depression, which was dependent on elevated comorbid, anxious arousal, and simultaneously, on low comorbid, anxious apprehension. These findings not only point to the importance of the frontal cortex in cognitive control but also highlight the important influence of comorbidity. The diversity of brain activity patterns observed in individuals with depression and anxiety might be used to match patients to specific interventions, because there is ever more research on the specificity of brain regions involved in treatment strategies for anxiety and depression (for a review, see Ressler \& Mayberg, 2007).

Research on frontal-cortex-related cognitive control processes (event-related potentials during modified Stroop task) indicates that these processes further deteriorate with the experience of successive depressive episodes, which is indicative of an increasing vulnerability (Vanderhasselt \& De Raedt, 2009). The framework proposed by De Raedt and Koster (2010) attempts to explain this increasing vulnerability after multiple episodes integrating the relation among HPA axis dysfunctions, frontal brain structures, and cognitive processes. In this framework, decreasing cognitive control processes are the crucial link between biological and cognitive factors. Understanding these interactions might also be important for the prediction of treatment outcomes. Using fMRI, Forbes et al. (2010) investigated treatment outcome prediction in adolescents with depression during a monetary reward task before an 8 -week open trial of CBT or of CBT combined with a selective serotonin reuptake inhibitor. Severity, anxiety symptoms, and depressive symptoms decreased over treatment, and the final levels of severity and anxiety symptoms were associated with pretreatment striatal reactivity. Moreover, rate of anxiety symptom reduction was associated with greater striatal reactivity and lower medial PFC reactivity. Surprisingly, however, decreases in depressive symptoms were not associated with reward-related brain function.

So far, we have discussed (1) subcortical (amygdala) sensitivity and a prefrontal failure to exert cognitive control and (2) the role of the ACC in the interaction between emotion and cognitive/behavioral control and in the relation between action monitoring and reward sensitivity as important features of depression. Cognitive control, however, likely consists of a number of different subprocesses, and it may be critical to understand which of these processes are impaired in depression and which may remain intact. A better understanding of the specific cognitive deficits that characterize depression may aid the development of theoretical models of this disorder and the development of effective interventions. Joormann, Nee, Berman, Jonides, and Gotlib (2010) investigated whether depression is associated with problems in controlling emotional content of short-term memory, which may result in sustained negative affect and vicious circles of negative thoughts. These authors used an experimental paradigm that allowed them to differentiate inhibitory dysfunctions in early and later stages of processing of emotional material. Depression was not associated with difficulties keeping irrelevant material from entering short-term memory, but it was associated with difficulty in removing irrelevant negative material from short-term memory, which was related to rumination. These findings may help us understand why some people recover easily from negative affect, whereas others initiate a vicious circle of increasingly negative ruminative thinking.

Closely investigating cognitive processes that may underlie rumination, Jones, Siegle, Muelly, Haggerty, and Ghinassi (2010) examined the degree to which depressed and never-depressed people who varied in trait rumination displayed different pupillary motility patterns during a cognitive task. Different frequencies of pupillary motility were used as an index of task-related processing versus intrinsically generated intrusive thoughts. The results suggested that engaging in intrinsic processing was related to rumination and could contribute to cognitive deficits observed in depression. Because prospective studies have shown that rumination plays a role in both the onset and maintenance of depression (Nolen-Hoeksema, 2000), trait rumination might be a key construct in understanding problems of nonresponse to treatment. One possibility is that a cognitive deficit in the emotion-attention con- 
trol network facilitates rumination, and changes in this network might decrease rumination; but rumination can also be conceived as a dysfunctional emotion-regulation strategy that people use. In line with Borkovec's (1994) suggestion that worry can be seen as a cognitive avoidance strategy, and given the overlap between worry and rumination, avoidance might be the mechanism underlying rumination in depression (De Raedt, 2006). Detailed representations or memories (e.g., traumatic past events) can be avoided by deliberately ruminating about one's depressed state in abstract terms (e.g., How should I go on with my life?). In the short term, the depressed individual does not become overwhelmed by strong emotions that are associated with these representations, but in the long term, this strategy backfires, since rumination undermines problem solving (Lyubomirsky \& Nolen-Hoeksema, 1993) and prevents a true confrontation, which may be necessary for healthy emotional processing (Foa \& Kozak, 1986). Thus, avoidance and difficulties in inhibiting can both be working mechanisms underlying rumination.

\section{Conclusion}

Traditionally, psychological theories and biological approaches have developed in relative isolation. Although these approaches were postulated within a biopsychosocial or a vulnerability-stress model, the precise nature of the interaction between biological and psychological factors is not well specified. The emergence of cognitive approaches to depression, emphasizing biased processing of emotional material at the level of memory, attention, and interpretation (e.g., Williams, Watts, MacLeod, \& Mathews, 1988), allows for an integration of insights from biological approaches and cognitive psychology within an affective neuroscience framework delineating vulnerability factors in depression. In this special issue, we present a selection of research from different perspectives based on an impressive variety of methodologies and theoretical frameworks that provide converging support for specific depression-related disturbances in neural circuitry involved in emotion generation and regulation. In this introductory article, we highlight that much of this information can be interpreted with regard to vulnerability factors that predispose to the onset, maintenance, and recurrence of depression.

An important treatment implication of the reviewed studies is the idea that it may be worthwhile to more directly target cognitive and neural processes in our interventions. Recent work on cognitive-bias modification, for example, has demonstrated that attentional biases can be trained and that this training leads to changes in mood and reduces reactivity to stressful events (MacLeod et al., 2002; Mathews \& MacLeod, 2002; Wadlinger \& Isaacowitz, 2008). On the basis of these findings, researchers have begun to examine attentional bias training with clinical samples, but no studies thus far have examined whether modifying attentional biases in depressed participants leads to improvements in emotion regulation.

Recently, studies have started to investigate the possibility of training cognitive control in depression and examining the effects of this training on emotion regulation (e.g., Joormann, Hertel, Brozovich, \& Gotlib, 2005). Siegle, Thompson, Carter, Steinhauer, and Thase (2007), for example, presented preliminary data demonstrating that a brief intervention targeted at increasing cognitive control in severely depressed outpatients led to significant decreases in both depressive symptoms and rumination. Indeed, recent work by this group suggests that training in attentional control may be an effective treatment component for depression (Siegle, Ghinassi, \& Thase, 2007). In this training, patients learn to selectively attend to certain sounds while ignoring irrelevant sounds. After receiving 2 weeks of this training, patients exhibited decreases in depressive symptoms, as compared with patients who received treatment as usual (Siegle, Ghinassi, $\&$ Thase, 2007). Notably, the training consisted of short sessions (15 $\mathrm{min}$ ) that used nonaffective stimuli, such as the sound of birds. This suggests that cognitive control can be improved with practice and further supports the hypothesis that individual differences in cognitive control may affect emotion regulation.

Finally, treatments based on recent research have begun to directly alter brain functioning using methods such as repetitive transcranial magnetic stimulation (rTMS) and neurofeedback. As an illustration of such translational research, it has been shown that multisession rTMS of the dorsolateral PFC can be effective as a treatment of severe depression by influencing brain activity and increasing attentional control (Leyman, De Raedt, Vanderhasselt, \& Baeken, in press; Vanderhasselt, De Raedt, Leyman, \& Baeken, 2009). Still, research that systematically examines the role of cognitive and biological factors for successful treatment and prevention of depression remains scarce, and we hope that this special issue will stimulate future research investigating these critical questions.

\section{AUTHOR NOTE}

This work was supported by Scientific Research Community (Automatic Processes in Psychopathology and Health-Related Behavior) Grant W000107N funded by the Research Foundation-Flanders (FWO). Correspondence concerning this article should be addressed to R. De Raedt, Ghent University, Department of Experimental, Clinical, and Health Psychology, Henri Dunantlaan 2, B-9000 Ghent, Belgium (e-mail: rudi .deraedt@ugent.be).

\section{REFERENCES}

BECK, A. T. (1963). Thinking and depression: 1. Idiosyncratic content and cognitive distortions. Archives of General Psychiatry, 9, 324-333.

Beck, A. T. (1976). Cognitive therapy and the emotional disorders. New York: International Universities Press.

BECK, A. T. (2008). The evolution of the cognitive model of depression and its neurobiological correlates. American Journal of Psychiatry, 165, 969-977.

Beevers, C. G., \& Carver, C. S. (2003). Attentional bias and mood persistence as prospective predictors of dysphoria. Cognitive Therapy \& Research, 27, 619-637.

Borkovec, T. D. (1994). The nature, functions, and origins of worry. In G. C. L. Davey \& F. Tallis (Eds.), Worrying: Perspectives on theory, assessment, and treatment (pp. 5-33). Chichester, U.K.: Wiley.

Browning, M., Holmes, E. A., \& Harmer, C. J. (2010). The modification of attentional bias to emotional information: A review of the techniques, mechanisms, and relevance to emotional disorders. Cognitive, Affective, \& Behavioral Neuroscience, 10, 8-20. 
Bush, G., LuU, P., \& Posner, M. I. (2000). Cognitive and emotional influences in anterior cingulate cortex. Trends in Cognitive Sciences, 4, 215-222.

Cuijpers, P., van Straten, A., Bohlmeijer, E., Hollon, S. D., \& ANDERSSON, G. (in press). The effects of psychotherapy for adult depression are overestimated: A meta-analysis of study quality and effect size. Psychological Medicine.

DE RAEDT, R. (2006). Activation of implicit self-schemas and a difficulty to disengage from negative cognitions in depression: An experimental psychopathology approach. Psychologica Belgica, 46, 117-130.

De RAedT, R., \& Koster, E. H. W. (2010). Understanding vulnerability for depression from a cognitive neuroscience perspective: A reappraisal of attentional factors and a new conceptual framework. Cognitive, Affective, \& Behavioral Neuroscience, 10, 50-70.

Dimidjian, S., Hollon, S. D., Dobson, K. S., Schmaling, K. B., KohlenberG, R. J., AdDIS, M. E., ET AL. (2006). Randomized trial of behavioral activation, cognitive therapy, and antidepressant medication in the acute treatment of adults with major depression. Journal of Consulting \& Clinical Psychology, 74, 658-670.

Ellenbogen, M. A., Carson, R. J., \& Pishva, R. (2010). Automatic emotional information processing and the cortisol response to acute psychosocial stress. Cognitive, Affective, \& Behavioral Neuroscience, 10, 71-82.

Engels, A. S., Heller, W., Spielberg, J. M., Warren, S. L., Sutton, B. P., BANich, M. T., \& Miller, G. A. (2010). Co-occurring anxiety influences patterns of brain activity in depression. Cognitive, Affective, \& Behavioral Neuroscience, 10, 141-156.

ENGLE, R. W. (2002). Working memory capacity as executive attention. Current Directions in Psychological Science, 11, 19-23.

FoA, E. B., \& KozAK, M. J. (1986). Emotional processing of fear: Exposure to corrective information. Psychological Bulletin, 99, 20-35.

Forbes, E. E., Olino, T. M., Ryan, N. D., Birmaher, B., Axelson, D., Moyles, D. L., \& DAHL, R. E. (2010). Reward-related brain function as a predictor of treatment response in adolescents with major depressive disorder. Cognitive, Affective, \& Behavioral Neuroscience, 10, 107-118.

Fu, C. H. Y., Williams, S. C. R., Cleare, A. J., Brammer, M. J., Walsh, N. D., KIM, J., ET AL. (2004). Attenuation of the neural response to sad faces in major depression by antidepressant treatment: A prospective, event-related functional magnetic resonance imaging study. Archives of General Psychiatry, 61, 877-889.

Gerra, G., Leonardi, C., Cortese, E., Zaimovic, A., Dell' Agnello, G., Manfredini, M., ET Al. (2009). Childhood neglect and parental care perception in cocaine addicts: Relation with psychiatric symptoms and biological correlates. Neuroscience \& Biobehavioral Reviews, 33, 601-610.

Goodwin, R. D., JACoBi, F., Bittner, A., \& Wittchen, H. U. (2006). Epidemiology of mood disorders. In D. J. Stein, D. J. Kupfer, \& A. F. Schatzberg (Eds.), Textbook of mood disorders (pp. 33-54). Washington, DC: American Psychiatric Publishing.

Gotlib, I. H., Krasnoperova, E., Yue, D. N., \& Joormann, J. (2004). Attentional biases for negative interpersonal stimuli in clinical depression. Journal of Abnormal Psychology, 113, 127-135.

HASHER, L., \& ZACKS, R. T. (1979). Automatic and effortful processes in memory. Journal of Experimental Psychology: General, 108, 356388.

Holmes, A. J., \& Pizzagalli, D. A. (2008). Spatiotemporal dynamics of error processing dysfunctions in major depressive disorder. $\mathrm{Ar}$ chives of General Psychiatry, 65, 179-188.

Holmes, A. J., \& Pizzagalli, D. A. (2010). Effects of task-relevant incentives on the electrophysiological correlates of error processing in major depressive disorder. Cognitive, Affective, \& Behavioral Neuroscience, 10, 119-128.

Ingram, R. E., Miranda, J., \& Segal, Z. V. (1998). Cognitive vulnerability to depression. New York: Guilford.

Jones, N. P., Siegle, G. J., Muelly, E. R., Haggerty, A., \& GhiNASSI, F. (2010). Poor performance on cognitive tasks in depression: Doing too much or not enough? Cognitive, Affective, \& Behavioral Neuroscience, 10, 129-140.

Joormann, J., Hertel, P. T., Brozovich, F., \& Gotlib, I. H. (2005). Remembering the good, forgetting the bad: Intentional forgetting of emotional material in depression. Journal of Abnormal Psychology, 114, 640-648.
Joormann, J., Nee, D. E., Berman, M. G., Jonides, J., \& Gotlib, I. H. (2010). Interference resolution in major depression. Cognitive, Affective, \& Behavioral Neuroscience, 10, 21-33.

Kirsch, I., Deacon, B. J., Huedo-Medina, T. B., Scoboria, A., Moore, T. J., \& Johnson, B. T. (2008). Initial severity and antidepressant benefits: A meta-analysis of data submitted to the Food and Drug Administration. PLoS Medicine, 5, e45.

Koole, S. L. (2009). The psychology of emotion regulation: An integrative review. Cognition \& Emotion, 23, 4-41.

Leyman, L., De Raedt, R., Schacht, R., \& Koster, E. H. W. (2007). Attentional biases for angry faces in unipolar depression. Psychological Medicine, 37, 393-402.

Leyman, L., De Raedt, R., Vanderhasselt, M. A., \& Baeken, C. (in press). Effects of repetitive transcranial magnetic stimulation of the dorsolateral prefrontal cortex on the attentional processing of emotional information in major depression: A pilot study. Psychiatry Research.

LyUbomirsky, S., \& Nolen-Hoeksema, S. (1993). Self-perpetuating properties of dysphoric rumination. Journal of Personality \& Social Psychology, 65, 339-349.

Macleod, C., Rutherford, E., Campbell, L., Ebsworthy, G., \& HolKer, L. (2002). Selective attention and emotional vulnerability: Assessing the causal basis of their association through the experimental manipulation of attentional bias. Journal of Abnormal Psychology, 111, 107-123.

Maheu, F. S., Dozier, M., Guyer, A. E., Mandell, D., Peloso, E., Poeth, K., ET AL. (2010). A preliminary study of medial temporal lobe function in youths with a history of caregiver deprivation and emotional neglect. Cognitive, Affective, \& Behavioral Neuroscience, 10, 34-49.

Masten, C. L., Guyer, A. E., Hodgdon, H. B., McClure, E. B., CharNEY, D. S., ERNST, M., ET AL. (2008). Recognition of facial emotions among maltreated children with high rates of post-traumatic stress disorder. Child Abuse \& Neglect, 32, 139-153.

Mathews, A., \& MacLeod, C. (2002). Induced processing biases have causal effects on anxiety. Cognition \& Emotion, 16, 331-354.

Mathews, A., \& MacLeod, C. (2005). Cognitive vulnerability to emotional disorders. Annual Review of Clinical Psychology, 1, 167-195.

Monk, C. S., Klein, R. G., Telzer, E. H., Schroth, E. A., ManNUZZA, S., Moulton, J. L., III, ET AL. (2008). Amygdala and nucleus accumbens activation to emotional facial expressions in children and adolescents at risk for major depression. American Journal of Psychiatry, 165, 90-98.

Nolen-Hoeksema, N. (2000). The role of rumination in depressive disorders and mixed anxiety/depressive symptoms. Journal of Abnormal Psychology, 109, 504-511.

Pereira, M. G., De Oliveira, L., Erthal, F. S., Joffily, M., MocaiBER, I. F., Volchan, E., \& Pessoa, L. (2010). Emotion affects action: Midcingulate cortex as a pivotal node of interaction between negative emotion and motor signals. Cognitive, Affective, \& Behavioral Neuroscience, 10, 94-106.

Phan, K. L., Wager, T. D., Taylor, S. F., \& Liberzon, I. (2004). Functional neuroimaging studies of human emotions. Central Nervous System Spectrums, 9, 258-266.

Phillips, M. L., Drevets, W. C., Rauch, S. L., \& Lane, R. (2003). Neurobiology of emotion perception II: Implications for major psychiatric disorders. Biological Psychiatry, 54, 515-528.

Pizzagalli, D. A., Dillon, D. G., Bogdan, R., \& Holmes, A. J. (in press). Reward and punishment processing in the human brain: Clues from affective neuroscience and implications for depression research. In O. Vartanian \& D. R. Mandel (Eds.), Neuroscience of decision making. New York: Psychology Press.

Pourtois, G., Spinelli, L., Seeck, M., \& Vuilleumier, P. (2010). Temporal precedence of emotion over attention modulations in the lateral amygdala: Intracranial ERP evidence from a patient with temporal lobe epilepsy. Cognitive, Affective, \& Behavioral Neuroscience, 10, 83-93.

Ressler, K. J., \& Mayberg, H. S. (2007). Targeting abnormal neural circuits in mood and anxiety disorders: From the laboratory to the clinic. Nature Neuroscience, 10, 1116-1124.

Siegle, G. J., Ghinassi, F., \& Thase, M. E. (2007). Neurobehavioral therapies in the 21st century: Summary of an emerging field and an extended example of cognitive control training for depression. Cognitive Therapy \& Research, 31, 235-262. 
Siegle, G. J., Thompson, W., Carter, C. S., Steinhauer, S. R., \& THASE, M. E. (2007). Increased amygdala and decreased dorsolateral prefrontal BOLD responses in unipolar depression: Related and independent features. Biological Psychiatry, 61, 198-209.

TAYlor, J. G., \& Fragopanagos, N. F. (2005). The interaction of attention and emotion. Neural Networks, 18, 353-369.

Turner, E. H., Matthews, A. M., Linardatos, E., Tell, R. A., \& Rosenthal, R. (2008). Selective publication of antidepressant trials and its influence on apparent efficacy. New England Journal of Medicine, 358, 252-260.

VAnderhasselt, M. A., \& De Raedt, R. (2009). Impairments in cognitive control persist during remission from depression and are related to the number of past episodes: An event related potentials study. Biological Psychology, 81, 169-176.
Vanderhasselt, M. A., De Raedt, R., Leyman, L., \& Baeken, C. (2009). Acute effects of repetitive transcranial magnetic stimulation on attentional control are related to antidepressant outcomes. Journal of Psychiatry \& Neuroscience, 34, 119-126.

WadLinger, H. A., \& IsaACowitz, D. M. (2008). Looking happy: The experimental manipulation of a positive visual attention bias. Emotion, 8, 121-126.

Williams, J. M. G., Watts, F. N., Macleod, C., \& Mathews, A. (1988). Cognitive psychology and emotional disorders. Chichester, U.K.: Wiley.

(Manuscript received September 21, 2009; revision accepted for publication October 28, 2009.) 